\title{
Plasmon-assisted quantum control of distant emitters
}

\author{
Cristian E. Susa ${ }^{\mathrm{a}}$, John H. Reina ${ }^{\mathrm{a}, \mathrm{b}}$, Richard Hildner ${ }^{\mathrm{c}}$ \\ ${ }^{a}$ Departamento de Física, Universidad del Valle, A.A. 25360, Cali, Colombia \\ ${ }^{b}$ Departamento de Óptica, Facultad de Física, Universidad Complutense, 28040 Madrid, Spain \\ ${ }^{c}$ Experimentalphysik IV, Universität Bayreuth, Universitätsstrasse 30, 95447 Bayreuth, Germany
}

\begin{abstract}
We show how to generate and control the correlations in a set of two distant quantum emitters coupled to a one-dimensional dissipative plasmonic waveguide. An external laser field enhances the dimer's steady-state correlations and allows an active control (switching on/off) of nonclassical correlations. The plasmon-assisted dipolar-interacting qubits exhibit persistent correlations, which in turn can be decoupled and made to evolve independently from each other. The setup enables long-distance ( 1 $\mu$ m) qubit control that works for both resonant and detuned emitters. For suitable emitter initialization, we also show that the quantum correlation is always greater than the classical one.
\end{abstract}

Keywords: Quantum information, Entanglement production and manipulation, Collective excitations, Coherent control of atomic interactions with photons, open systems; quantum statistical methods

PACS: 03.67.-a, 03.67.Bg, 73.20.Mf, 32.80.Qk, 03.65.Yz

\section{Introduction}

The construction of large quantum networks with controllable long-distance coupling of qubits is one of the key goals in quantum information science [1]. To generate the required qubit-qubit correlations (microwave or optical) photons are typically used. Recently, a novel approach based on single quantum emitters coupled to one-dimensional plasmonic waveguides has been proposed, and entanglement of qubits mediated by surface plasmons in such a setup has been explored [2, 3]. Surface plasmons are collective excitations that have become an important physical resource in many applications in physics, chemistry, and materials science [4, 5]. In particular, the coupling of single emitters to plasmonic structures [6, 7, 8] has attracted substantial attention, because it allows the manipulation of the emission properties as well as the enhancement of the interaction between quantum emitters in the vicinity of these structures [9, 10, 11].

Non-local quantum correlations and entanglement in quantum systems under the action of decoherence effects have been arduously investigated not only because of their fundamental physical implications (see e.g., [12, 13]), but also because of their utmost relevance to the development of novel quantum technologies [1]. The influence of the system-bath coupling over the reduced system's quantum correlations has been studied in different physical systems such as quantum dots [14], superconducting qubits [15], atoms and photons [16], and biomolecular systems [17], to cite just but a few. We have recently reported on the distribution of classical and quantum correlations that arise in a bipartite emitter system coupled to a \footnotetext{
Reina)

Email address: john.reina@correounivalle. edu . co (John H.
}

Preprint submitted to Physics Letters A plasmonic waveguide [18]. We have shown that such quantum correlations are more robust through the dissipative dynamics than the classical ones, for several experimentally accessible scenarios, and that the emitters collective properties that arise from the interaction with the plasmons allow an additional degree of quantum control on the correlations dynamics [18, 19].

In this Letter, we give a protocol for actively enabling a plasmon-assisted long-distance (about $1 \mu \mathrm{m}$ ) qubit conditional dynamics between emitters which are externally-driven by a coherent laser field. Such a quantum mechanism works for both resonant and detuned qubits, and is optimized by laser pumping and by tailoring the separation between the emitters. Moreover, from the dimer's conditional entropy, we analytically identify specific conditions for which the quantum correlation is always greater than the classical correlation (cf. [20, 21, 22]).

In section 2 we introduce the quantifiers of quantum and classical correlations, and the corresponding quantum master equation used to describe the emitters' dissipative dynamics is given in section 3. The quantum control that arises from the correlations dynamics is set for: resonant emitters without laser excitation (section 4), resonant emitters under the action of a coherent laser field (section 5), and for emitters with different transition energies (section 6). Concluding remarks are given in section 7

\section{Quantum correlations}

The quantum mutual information $\mathcal{I}\left(\rho_{A B}\right)=S\left(\rho_{A}\right)+S\left(\rho_{B}\right)-$ $S\left(\rho_{A B}\right)$ gives a measure of the total correlation in a bipartite qubit state $\rho_{A B}[22,[23,24]$, and this may be separated into purely quantum- $-Q\left(\rho_{A B}\right)$ (e.g., via the quantum discord

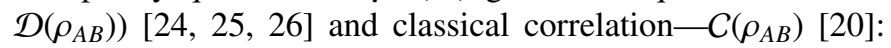
$\mathcal{I}\left(\rho_{A B}\right)=Q\left(\rho_{A B}\right)+C\left(\rho_{A B}\right)$. The details of calculation of $C$ 
and $Q$ are left to the appendix. The von Neumann entropy $S(\rho)=-\operatorname{Tr} \rho \log _{2} \rho$, and $\rho_{A(B)}=\operatorname{Tr}_{B(A)} \rho_{A B}$ is the reduced density operator of the partition $A(B)$. Discord has been linked to the computational speedup in an efficient model of quantum computation [27] and has been pinpointed as a valuable resource in quantum information protocols [28, 27].

Although Lindblad conjectured that, for any quantum state, $C(\rho) \geq Q(\rho)[22,20,21]$, we give an entropy condition for which the quantum correlation is always greater than the classical one [29, 19]: since $\mathcal{I}=Q+C$, we ask if the inequality $\mathcal{I}-2 C \geq 0$ is ever met. The sought entropy bound reads

$$
2 \mathcal{S}\left(\rho_{A \mid \Pi_{j}^{B}}\right)-S\left(\rho_{A B}\right)+S\left(\rho_{B}\right)-S\left(\rho_{A}\right) \geq 0,
$$

where $\mathcal{S}\left(\rho_{A \mid \Pi_{j}^{B}}\right)=\min _{\left\{\Pi_{j}^{B}\right\}}\left\{\sum_{j} p_{j} S\left(\rho_{A \mid \Pi_{j}^{B}}\right)\right\}, S\left(\rho_{A \mid \Pi_{j}^{B}}\right)$ is the entropy associated to the density matrix of subsystem $A$ after the measure. Equation (1) is indeed satisfied, at all times, for suitable resonant emitters.

To distinguish the quantum correlations that arise for entangled states and separable states, we quantify the emitters' entanglement via the entanglement of formation $\mathcal{E}_{\mathcal{F}}$. For twoqubit systems, $\mathcal{E}_{\mathcal{F}}\left(\rho_{A B}\right)=\varepsilon\left(\left[1+\sqrt{1-C^{2}\left(\rho_{A B}\right)}\right] / 2\right)$, where $\varepsilon(r)=-r \log _{2} r-(1-r) \log _{2}(1-r)$ denotes the binary entropy function, and the concurrence $C\left(\rho_{A B}\right)=\max \left\{0, \lambda_{1}-\lambda_{2}-\lambda_{3}-\lambda_{4}\right\}$, where the $\lambda_{i}$ 's are, in decreasing order, the eigenvalues of the matrix $\sqrt{\rho_{A B} \tilde{\rho}_{A B}} ; \tilde{\rho}_{A B}=\left(\sigma_{y} \otimes \sigma_{y}\right) \bar{\rho}_{A B}\left(\sigma_{y} \otimes \sigma_{y}\right), \bar{\rho}_{A B}$ is the elementwise complex conjugate of $\rho$, and $\sigma_{y}$ is the Pauli matrix [30]. This entanglement metric is of entropic character and can be compared on the same grounds with the discord [18].

\section{Plasmon-emitter master equation}

We consider a pair of distant emitters that act as a dipoledipole two-qubit system via the interaction with the plasmon modes in a metallic nanostructure. The total Hamiltonian of the system plus environment can be written as ( $\hbar$ is the reduced Planck's constant):

$$
\begin{aligned}
\hat{H}= & \hat{H}_{S}+\hat{H}_{E}+\hat{H}_{i n t}=\sum_{i=1}^{2} \frac{1}{2} \hbar \omega_{i} \hat{\sigma}_{z}^{(i)}+ \\
& +\int d^{3} \boldsymbol{r} \int_{0}^{\infty} d \omega \hbar \omega \hat{f}_{\omega}^{\dagger}(\boldsymbol{r}) \hat{f}_{\omega}(\boldsymbol{r})-\sum_{i=1}^{2} \hat{\mu}_{i} \cdot \hat{E}\left(\boldsymbol{r}_{i}\right),
\end{aligned}
$$

where the first two terms denote the free energy of the twoqubit system and the free energy of the electromagnetic field represented by a bosonic bath, respectively; $\hat{\sigma}_{z}^{(i)}$ is the $z$ Pauli matrix associated to the $i$-th emitter of transition frequency $\omega_{i}$, and $\hat{f}_{\omega}(\boldsymbol{r})$, and $\hat{f}_{\omega}^{\dagger}(\boldsymbol{r})$ are the bosonic excitation operators of the quantized electromagnetic field with the usual commutation relations $\left[\hat{f}_{\omega}(\boldsymbol{r}), \hat{f}_{\omega^{\prime}}^{\dagger}\left(\boldsymbol{r}^{\prime}\right)\right]=\delta\left(\omega-\omega^{\prime}\right) \delta\left(\boldsymbol{r}-\boldsymbol{r}^{\prime}\right)$, and $\left[\hat{f}_{\omega}(\boldsymbol{r}), \hat{f}_{\omega^{\prime}}\left(\boldsymbol{r}^{\prime}\right)\right]=\left[\hat{f}_{\omega}^{\dagger}(\boldsymbol{r}), \hat{f}_{\omega^{\prime}}^{\dagger}\left(\boldsymbol{r}^{\prime}\right)\right]=0$. The last term represents the interaction between the dipole operator $\hat{\mu}=\mu_{i} \hat{\sigma}_{+}^{(i)}+\mu_{i}^{*} \hat{\sigma}_{-}^{(i)}$ and the quantized field operator $\hat{E}(\boldsymbol{r})=\hat{E}^{+}(\boldsymbol{r})+$ H.c., where $\sigma_{+}^{(i)}=\left|1_{i}\right\rangle\left\langle 0_{i}\right|\left(\sigma_{-}^{(i)}=\left|0_{i}\right\rangle\left\langle 1_{i}\right|\right)$ are the raising (lowering) Pauli operators acting on emitter $i(|0\rangle$ and $|1\rangle$ denote the ground and first excited state which represent the qubit computational basis), and $\hat{E}^{+}(\boldsymbol{r})=\int_{0}^{\infty} d \omega \hat{E}(\boldsymbol{r}, \omega)$ is the positive frequency part,

$$
\hat{E}(\boldsymbol{r}, \omega)=\mathrm{i} \sqrt{\frac{\hbar}{\pi \epsilon_{0}}} \frac{\omega^{2}}{c^{2}} \int d^{3} \boldsymbol{r}^{\prime} \sqrt{\epsilon^{\prime \prime}\left(\boldsymbol{r}^{\prime}, \omega\right)} \boldsymbol{G}\left(\boldsymbol{r}, \boldsymbol{r}^{\prime}, \omega\right) \hat{f}_{\omega}\left(\boldsymbol{r}^{\prime}\right) .
$$

The Green's tensor $\boldsymbol{G}\left(\boldsymbol{r}, \boldsymbol{r}^{\prime}, \omega\right)$ satisfies the Maxwell-Helmholtz wave equation, supports the electromagnetic interaction from $\boldsymbol{r}^{\prime}$ to $\boldsymbol{r}$, and contains all the information about the coherent and incoherent properties of the system, i.e., about the dipole-dipole shift and the different channels of radiation through the vacuum (far field) and through the metal. $\epsilon^{\prime \prime}(\boldsymbol{r}, \omega)$ is the imaginary part of the electric permittivity of the metal, and this is considered as a constant value corresponding to the permittivity of the silver at the operational wavelength described here, and $\epsilon_{0}$ is the permittivity of the vacuum.

The dynamics of the total system (emitters plus electromagnetic field) can be derived in the Schrödinger picture from the Liouville-von Neumann equation $\dot{\rho}_{S-E}=-\frac{i}{\hbar}\left[\hat{H}, \rho_{S-E}\right]$, which, in the interaction picture (denoted by the superscript $I$ ), can be written as the following integro-differential equation

$$
\begin{aligned}
\dot{\rho}_{S-E}^{I}(t)= & -\frac{\mathrm{i}}{\hbar}\left[\hat{H}_{i n t}^{I}(t), \rho_{S-E}^{I}(0)\right]- \\
& -\frac{1}{\hbar^{2}} \int_{0}^{t} d t^{\prime}\left[\hat{H}_{i n t}^{I}(t),\left[\hat{H}_{i n t}^{I}\left(t^{\prime}\right), \rho_{S-E}^{I}\left(t^{\prime}\right)\right]\right],
\end{aligned}
$$

where $\rho_{S-E}^{I}(t)=e^{\mathrm{i}\left(\hat{H}_{S}+\hat{H}_{E}\right) t / \hbar} \rho_{S-E} e^{-\mathrm{i}\left(\hat{H}_{S}+\hat{H}_{E}\right) t / \hbar}$, and $\hat{H}_{\text {int }}^{I}(t)=$ $e^{\mathrm{i}\left(\hat{H}_{S}+\hat{H}_{E}\right) t / \hbar} \hat{H}_{\text {int }} e^{-\mathrm{i}\left(\hat{H}_{S}+\hat{H}_{E}\right) t / \hbar}$. Here, we consider atom-like (small) emitters with an operational wavelength in the optical frequency regime (we use $\lambda_{0}=640 \mathrm{~nm}$ ) coupled to a broadband plasmonic waveguide. We perform the Born-Markov approximation $\left(\rho_{S-E}^{I}\left(t^{\prime}\right)=\rho^{I}\left(t^{\prime}\right) \otimes \rho_{E}(0)\right.$, and $\left.\rho^{I}\left(t^{\prime}\right) \rightarrow \rho^{I}(t)\right)$ in order to solve Eq. (3) due to the weak coupling between the emitters and the plasmonic electromagnetic field [2, 3, 11, 31]. Additionally, we avoid rapidly oscillating terms much higher than $\omega_{i}$ by applying the rotating wave approximation (RWA) to the interaction part of the Hamiltonian in Eq. (3), such that $\hat{H}_{i n t}=-\boldsymbol{\mu}_{1} \sigma_{+}^{(1)} \hat{E}^{\dagger}\left(\boldsymbol{r}_{1}\right)-\boldsymbol{\mu}_{2} \sigma_{+}^{(2)} \hat{E}^{\dagger}\left(\boldsymbol{r}_{2}\right)+$ H.c..

Tracing out Eq. (3) over the environment degrees of freedom, and going back to the Schrödinger picture we can, under the above assumptions, describe the dimer's dissipative dynamics by means of the following quantum master equation [11, 19, 32]

$$
\dot{\rho}=\frac{i}{\hbar}\left[\rho, \hat{H}_{\mathrm{eff}}\right]-\sum_{i, j=1}^{2} \frac{\Gamma_{i j}}{2}\left(\rho \sigma_{+}^{(i)} \sigma_{-}^{(j)}+\sigma_{+}^{(i)} \sigma_{-}^{(j)} \rho-2 \sigma_{-}^{(i)} \rho \sigma_{+}^{(j)}\right),
$$

where the effective dimer's Hamiltonian $\hat{H}_{\text {eff }}=\hat{H}_{S}+\hat{H}_{12}+\hat{H}_{L}$ contains the coherent dipole-dipole shift $\hat{H}_{12}=\frac{1}{2} \hbar V\left(\sigma_{x}^{(1)} \otimes\right.$ $\sigma_{x}^{(2)}+\sigma_{y}^{(1)} \otimes \sigma_{y}^{(2)}$ ), and the laser-qubit interaction $\hat{H}_{L}$ (see section 5. The strength of the effective dipole-dipole interaction is calculated as:

$$
V=\frac{1}{\pi \epsilon_{0} c^{2} \hbar} \mathcal{P} \int_{0}^{\infty} d \omega \frac{\omega^{2} \operatorname{Im}\left[\boldsymbol{\mu}_{1}^{*} \boldsymbol{G}\left(\omega, \mathbf{r}_{1}, \mathbf{r}_{2}\right) \boldsymbol{\mu}_{2}\right]}{\omega-\omega_{0}},
$$


where $\omega_{0}=\left(\omega_{1}+\omega_{2}\right) / 2$ and $\mathcal{P}$ denotes the principal part of the integral. The second term of Eq. (4) accounts for the dimer's incoherent effects that arise from radiation into the vacuum, dissipation through losses in the metal, and the excitation of the surface plasmons that propagate on the metallic surface [3, 33]. If the losses in the metal are negligible, the two remaining dissipative channels to be accounted for are captured by

$$
\Gamma_{i j}=\frac{2 \omega_{0}^{2}}{\epsilon_{0} c^{2} \hbar} \operatorname{Im}\left[\boldsymbol{\mu}_{i}^{*} \boldsymbol{G}\left(\omega_{0}, \mathbf{r}_{i}, \mathbf{r}_{j}\right) \boldsymbol{\mu}_{j}\right],
$$

where $\Gamma_{11}=\Gamma_{22} \equiv \Gamma$, and $\Gamma_{12}=\Gamma_{21}^{*} \equiv \gamma$ are the individual and collective spontaneous emission rates, respectively [32], and $i, j=1,2$. The emitters-plasmon dissipative coupling renders new dipole-dipole strengths $V$ and collective damping rates $\gamma$. If we consider that the emitters' dipoles are equally oriented and are located at the same distance to the surface of the metal, and that the emitters' most relevant dissipative contribution is due to the plasmon modes, an exact calculation of $V$ (Eq. (5) ) and $\gamma$ (Eq. [6) follows from the Green's functions of the plasmon excitations [2, 3, 9, 11]:

$$
V=\frac{\Gamma}{2} \beta e^{-\frac{\lambda_{p l}}{2 L} \zeta} \sin (2 \pi \zeta) ; \quad \gamma=\Gamma \beta e^{-\frac{\lambda_{p l}}{2 L} \zeta} \cos (2 \pi \zeta)
$$

where the wavelength of plasmons $\lambda_{p l} \equiv 2 \pi / k_{p l}, k_{p l}$ is the plasmon wave vector, $\zeta \equiv d / \lambda_{p l}, d$ gives the distance between emitters, and $L$ is the propagation length of the plasmons.

Equations (7) are valid for high $\beta$-factors $\left(\beta \equiv \Gamma^{\text {guided }} / \Gamma\right.$, $\Gamma=\Gamma^{\mathrm{vac}}+\Gamma^{\text {guided }}$, which means that the dimer's main dissipative mechanism is due to the propagating plasmons on the metal surface, and hence the fraction of emitted radiation by the plasmonic propagating mode $\beta \mapsto 1$. This factor depends on the vertical distance between the emitters and the metallic surface. Here, we consider geometries like $\Lambda$-wedge or $V$-groove as our plasmonic waveguides, for which the plasmonic excitations have been shown to substantially enhance the interqubit interaction [9, 10], thus validating Eqs. (7). In particular, for the $V$-groove channel, $\beta$-factors $\sim 0.91$ (and higher) are expected for an emitter-metal vertical separation above the bottom of the $V$-channel waveguide of about $160 \mathrm{~nm}$, and a depth of the channel of $140 \mathrm{~nm} \mathrm{[2,} \mathrm{3,} \mathrm{9].} \mathrm{For} \mathrm{such} \mathrm{a} \mathrm{channel} \mathrm{depth,}$ we note that shorter emitter-metal distances $(<80 \mathrm{~nm})$ imply a reduction in the $\beta$-factor since the losses into the metal become appreciable. On the other hand, larger emitter-metal distances ( $>180 \mathrm{~nm}$ ), also diminish the $\beta$-factor since radiation into the vacuum becomes the major decay contribution [3]. In addition, the 'plasmonic approximation' given by Eqs. (7) works well for emitter-emitter separations larger than $\sim \lambda_{\mathrm{pl}} / 4$ [3, 11], and in this case $|\gamma| \leq \Gamma$ and $|V| \leq \frac{\Gamma}{2}$. Below such distance, the vacuum electromagnetic fluctuations become more significant and modify the collective parameters in equations (5) and (6).

A general two-qubit state can be written as $\epsilon=\frac{1}{4}\left(I_{4 \times 4}+\vec{a} \vec{\sigma} \otimes\right.$ $\left.I_{2 \times 2}+I_{2 \times 2} \otimes \vec{b} \vec{\sigma}+\sum_{i=1}^{3} h_{i} \sigma_{i} \otimes \sigma_{i}\right)$, where $\vec{a}=\left(a_{1}, a_{2}, a_{3}\right), \vec{b}=$ $\left(b_{1}, b_{2}, b_{3}\right) \in \mathbb{R}^{3}$, and $h_{i} \in \mathbb{R}[26,34]$. The initial density matrix used in our calculations adopts an $X$-like structure with $\vec{a}=$

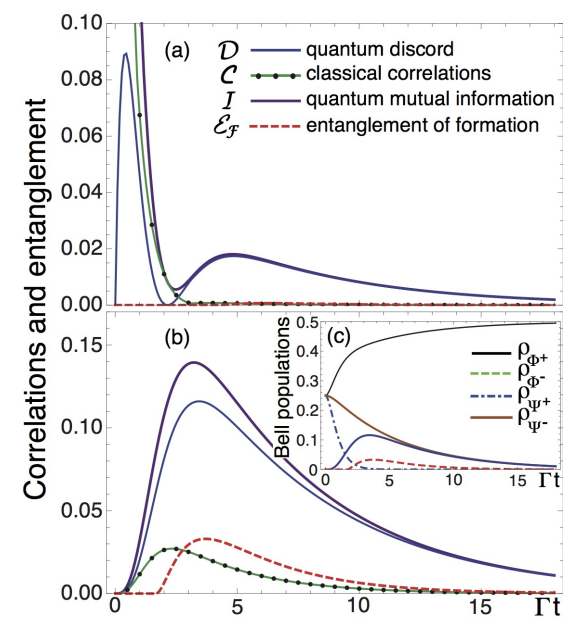

Figure 1: Dynamics of correlations for initial states (a) $\rho_{C}$, and (b) $\rho_{M M}$. We employ optimal plasmonic waveguide parameters [2]: $L=2 \mu \mathrm{m}, \beta=0.94$, $\lambda_{p l}=542 \mathrm{~nm}$ (operational wavelength $\lambda_{0}=640 \mathrm{~nm}$ ). The emitters are separated a distance $d=\lambda_{p l}(\zeta=1)$. (c) Bell states populations for the case (b).

$$
\begin{aligned}
\left(0,0, a_{3}\right), \vec{b}= & \left(0,0, b_{3}\right), \text { and } h_{1}=h_{2}=\eta \\
\rho_{A B}(0) & =\left(\begin{array}{cccc}
\frac{1+c_{+}+h_{3}}{4} & 0 & 0 & 0 \\
0 & \frac{1+c_{-}-h_{3}}{4} & \frac{\eta}{2} & 0 \\
0 & \frac{\eta}{2} & \frac{1-c_{-}-h_{3}}{4} & 0 \\
0 & 0 & 0 & \frac{1-c_{+}+h_{3}}{4}
\end{array}\right),
\end{aligned}
$$

where $c_{ \pm}=a_{3} \pm b_{3}$, and $a_{3}, b_{3}, \eta$, and $h_{3}$ are chosen such that $\rho_{A B}(0)$ is a well-defined density matrix [34].

\section{Resonant molecules without laser pumping}

For the above initial state, and identical emitters with transition frequencies $\omega_{1}=\omega_{2} \equiv \omega_{0}$, Eq. (4) admits an analytical solution in the absence of optical driving. The non-trivial density matrix elements read $a(t) \equiv \rho_{11}=1-b_{+}(t)-b_{-}(t)-f(t)$, where $f(t) \equiv \rho_{44}=\frac{1}{4} p e^{-2 \Gamma t}$, with $p=1+h_{3}-c_{+}$, and

$$
\begin{aligned}
& b_{ \pm}(t) \equiv\left\{\begin{array}{c}
\rho_{22}^{+} \\
\rho_{33}^{-}
\end{array}\right\}= \\
& \frac{e^{-\Gamma t}}{4 \gamma_{-}^{2}}\left[-p \gamma_{+}^{2} e^{-\Gamma t}-\left(c_{+} \gamma_{+}^{2}-2\left(\Gamma^{2}+h_{3} \gamma^{2}\right)\right) \cosh (\gamma t)-\right. \\
& \left.2\left(p \gamma \Gamma+\eta \gamma_{-}^{2}\right) \sinh (\gamma t) \pm c_{-} \gamma_{-}^{2} \cos (2 V t)\right] \\
& z(t) \equiv \rho_{23}= \\
& \frac{e^{-\Gamma t}}{4 \gamma_{-}^{2}}\left[-2 p \gamma \Gamma e^{-\Gamma t}+2\left(\eta \gamma_{-}^{2}+p \gamma \Gamma\right) \cosh (\gamma t)+\right. \\
& \left.\left(c_{+} \gamma_{+}^{2}-2\left(\Gamma^{2}+h_{3} \gamma^{2}\right)\right) \sinh (\gamma t)+\mathrm{i} c_{-} \gamma_{-}^{2} \sin (2 V t)\right]
\end{aligned}
$$

and $z^{*}(t) \equiv \rho_{32}$, with $\gamma_{ \pm}^{2}=\Gamma^{2} \pm \gamma^{2}$. Importantly, the initial states $\rho(0)$ contain the eigenstates of the system's Hamiltonian $\left(\hat{H}_{S}+\hat{H}_{12}\right):|00\rangle,\left|\Psi^{ \pm}\right\rangle=\frac{1}{\sqrt{2}}(|01\rangle \pm|10\rangle),|11\rangle$, which allows for their experimental generation, control, and read-out [35, 36].

From Eqs. (8) we see that for $c_{-}=0$ the density matrix becomes independent of the dipolar interaction $V\left(b_{+}=b_{-}=b\right)$ 

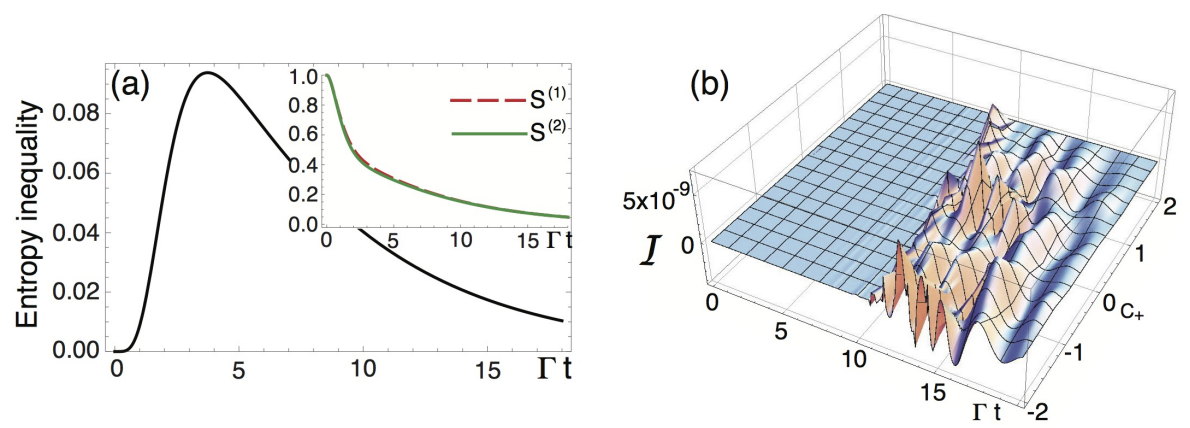

Figure 2: (a) Left-hand side of inequality Eq. 1), for the case shown in Fig. 1 b). The inset shows the evolution of the quantities $S^{(1)}$ (dashed red curve) and $S^{(2)}$ (solid green curve), whose minima give the conditional entropy. (b) Numerical simulation of the quantum mutual information for the set of initial states $\Xi:=\left\{c_{-}=0, \eta=0, h_{3}=c_{+}^{2} / 4\right\}$ in Eqs. 8]. The same result is obtained for the other correlations.

and, as such, the quantum correlation dynamics is only due to the collective damping $\gamma$. Such correlations persist for long times and for a large emitters' separation $\left(d=\lambda_{p l} ; \zeta=1\right)$, as shown in Fig. 1. For the plasmon waveguide we used realistic parameters $L=2 \mu \mathrm{m}, \beta=0.94$, and $\lambda_{p l} \approx 542 \mathrm{~nm}$, that follows from the dispersion relation reported in Ref. [2], based on an operational wavelength $\lambda_{0}=640 \mathrm{~nm}$. These parameters can be achieved with e.g. plasmonic wedges $(\Lambda)$ and V-groove waveguides. Although we have chosen $\zeta=1$, which makes $V=0$, our results are more general and can be extrapolated to other $\zeta$ values because the considered initial states evolve completely independently of $V$.

Figure 1 (a) shows the correlations dynamics for the classically correlated initial state $\rho_{C}=\frac{1}{2}|00\rangle\left\langle 00\left|+\frac{1}{2}\right| 11\right\rangle\langle 11|$ for which $C=\mathcal{I}=1$. Further, in Fig. 1 . b) we show how the correlations are increased for a completely uncorrelated initial state $\rho_{M M}=\operatorname{diag}\left\{\frac{1}{4}, \frac{1}{4}, \frac{1}{4}, \frac{1}{4}\right\}$. Note that while the quantum discord increases from $t=0$, the emitters follow entanglement sudden birth [37] for $t \sim 4 / \Gamma$ (Fig. 1](a)), and $t \sim 2 / \Gamma$ (Fig. 11.b)). For $t>10 / \Gamma$ Fig. 11(a) shows that the system becomes disentangled, quantum discord, however, equals $\mathcal{I}$ and the nonclassical correlation represents the total correlation in the dimer (i.e., $C=0$ ). Figure 1 (c) plots the population dynamics of the Bell basis states $\rho_{\Psi^{ \pm}} \equiv\left|\Psi^{ \pm}\right\rangle\left\langle\Psi^{ \pm}\right|$and $\rho_{\Phi^{ \pm}},\left|\Phi^{ \pm}\right\rangle=\frac{1}{\sqrt{2}}(|00\rangle \pm|11\rangle)$, as well as the time evolution of discord and entanglement for $\rho(0)=\frac{1}{4}\left(\rho_{\Psi^{+}}+\rho_{\Psi^{-}}+\rho_{\Phi^{+}}+\rho_{\Phi^{-}}\right)$[Fig. 1 (b)]: although the entanglement of formation $\left(\mathcal{E}_{\mathcal{F}}\right)$ eventually vanishes, the dynamics of $\mathcal{D}$ quickly approaches that of the antisymmetric Bell state $\rho_{\Psi^{-}}$, which decays at the slowest rate $\Gamma-\gamma$; this is due to the natural mixture of the Bell populations which spontaneously create quantum correlations beyond entanglement, here captured by discord. Figure 1 thus shows that, for suitable (unentangled) initialization of the emitters, discord is the most robust and persistent nonclassical correlation available.

An important point to note is that for the initial state $\rho_{M M}$ $\left(c_{-}=0\right)$ the quantum correlation is always greater than the classical correlation. Under these initial conditions the inequality given by Eq. (1) is satisfied; they have an associated condi- tional entropy $\mathcal{S}\left(\rho_{A \mid \Pi_{j}^{B}}\right)=\min _{\left\{\Pi_{j}^{B}\right\}}\left(S^{(1)}, S^{(2)}\right)$, with

$$
\begin{aligned}
S^{(1)=} & -a \log _{2} \frac{a}{a+b}-b \log _{2} \frac{b}{a+b}- \\
& b \log _{2} \frac{b}{b+f}-f \log _{2} \frac{f}{b+f}, \\
S^{(2)=} & -\frac{1}{2}(1-\xi) \log _{2} \frac{1}{2}(1-\xi)-\frac{1}{2}(1+\xi) \log _{2} \frac{1}{2}(1+\xi),
\end{aligned}
$$

where $\xi^{2}=(a-f)^{2}+|z|^{2}$, the marginals $S\left(\rho_{A}\right)=S\left(\rho_{B}\right)$, and we arrive at $2 \mathcal{S}\left(\rho_{A \mid \Pi_{j}^{B}}\right)-S\left(\rho_{A B}\right) \geq 0$. A direct calculation of $S^{(1)}$ and $S^{(2)}$ then demonstrates that the quantum correlation is greater than the classical one, as can be seen in Fig.2(a), where we have plotted the entropy bound (Eq. (1)). This behaviour has also been demonstrated for other emitters' preparations [18].

If we now choose the emitters' mutual separation such that $\zeta_{n}=\frac{1}{4}(2 n+1), n$ integer, the damping $\gamma$ vanishes according to Eqs. (7). As the emitters' dynamics is independent of $V$, the density matrix elements read

$$
\begin{aligned}
f(x) & =\frac{1}{4} p x^{2} ; \quad z(x)=\frac{1}{2} \eta x, \\
b_{ \pm}(x) & =\frac{1}{4} x\left[2\left(1-\frac{c_{+}}{2}\right)-p x\right], \\
a(x) & =1+\frac{1}{4} x\left[p x+4\left(\frac{c_{+}}{2}-1\right)\right],
\end{aligned}
$$

where $x \equiv e^{-\Gamma t}$. For this family of initial states (which includes the eigenstates of $\hat{H}_{\text {eff }}$ in absence of the coherent external laser), the dynamics of all correlations has an asymptotic decay following the time parameter $x$, in contrast to the scenario shown in Fig. 1 for which $\gamma$ is non-zero. If we add the conditions $\eta=0$ and $h_{3}=c_{+}^{2} / 4$, the density matrix adopts a diagonal structure without quantum correlations. This also allows us to show that $\rho_{A B}=\rho_{A} \otimes \rho_{B}$, and hence $C=0$. Since $\mathcal{I}\left(\rho_{A B}\right)$ gives the relative entropy between the matrices $\rho_{A B}$ and $\rho_{A} \otimes \rho_{B}$, it can also be seen that $\mathcal{I}\left(\rho_{A B}\right)=0$, and hence there are neither quantum nor classical correlations for this time evolution. Figure 2 (b) shows the numerical simulation for the quantum mutual information as a function of the parameter $c_{+}$(the fluctuations around $\sim 10^{-9}$ are due to numerical errors and do not imply $\mathcal{I} \neq 0$ ). Thus the system evolves through a path of product states even though $V \neq 0$. This provides a useful method for controlling the dynamics of correlations in two-qubit systems. 

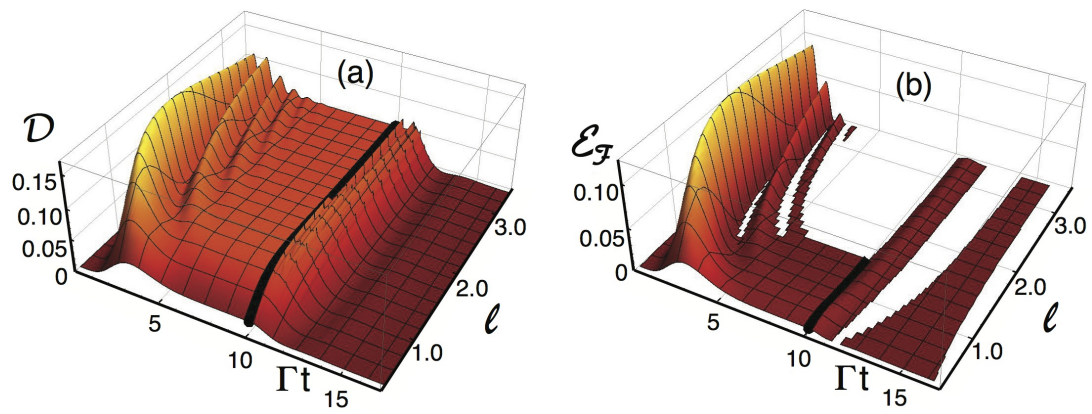

Figure 3: (a) Quantum discord and (b) entanglement of formation as functions of the laser field amplitude $\ell$, for resonant qubits $\omega_{L}=\omega$, and initial (ground) state $|00\rangle$. Waveguide parameters are as in Fig. $1, d=3 \lambda_{p l} / 4$, so $\gamma=0$. The black solid curve indicates the time at which the laser is turned off.

\section{Resonant molecules under laser pumping}

Illumination with a laser field, in conjunction with the emitters-plasmon coupling, allows a reestablishment of the correlations, which were lost due to the $V$-independence of the matrix elements. The light-emitter interaction is given by $\hat{H}_{L}=$ $\sum_{i} \hbar \ell_{i}\left(\sigma_{-}^{(i)} e^{\mathrm{i} \omega_{L} t}+\sigma_{+}^{(i)} e^{-\mathrm{i} \omega_{L} t}\right)$, with $\hbar \ell_{i}=-\hat{\mu}_{i} \cdot \hat{E}_{i}^{(L)}, i=1,2[32]$.

To illustrate this mechanism, we consider two individual molecules initially in their ground states, with resonance wavelength $\lambda_{0}$ in order to reach the optimal waveguide parameters of Fig. 11 We now, however, consider a shorter emitters' separation: $\zeta_{1}=3 / 4$, such that $\gamma=0$. The chosen values for the waveguide parameters at $\lambda_{0}$ simulate structures such as plasmonic wedges $(\Lambda)$ and $\mathrm{V}$-groove waveguides, which have been theoretically demonstrated to exhibit the greatest plasmon mode propagation [2, 9, 10]. Figure 3 depicts the evolution of the quantum discord (graph (a)) and the entanglement of formation (graph (b)) as functions of time (in $1 / \Gamma$ units), and laser intensity, under the choice $\ell_{1} \equiv \ell \gg \ell_{2}$. As an illustration, we fix $\ell_{2}=0$ and $\ell \in[0.4 \Gamma, 3.6 \Gamma]$ such that the effect of the coherent light over the emitter 1 is much stronger than that over emitter 2 (the latter being negligible). This particular choice is meant to highlight our main results and does not imply any restriction on the validity of the mechanism here presented or loss of generality for other choices of laser intensity.

By turning on the laser field (at $t=0$ ) to illuminate emitter 1 with an intensity $\ell$, we reach a time regime for which the correlations dynamics stabilizes $(\mathcal{D} \sim 0.06)$, as conservatively indicated by the thick black curve in Fig. 3 at $\Gamma t \sim 10$. If we then turn off the laser field at $\Gamma t=10$, the emitters decay again to the ground state well before $\Gamma t \sim 15$. The conditional dynamics between the laser field and the dipolar interaction favours such a behaviour. We point out that if, in the same scenario, the dipole-dipole interaction were zero (non-interacting qubits or emitters in vacuum with interqubit separation $d=3 \lambda / 4$ ), the correlations would then equal zero even if the laser were turned on (not shown). We have also checked that the scheme works for other input states which satisfy the initial condition $\Xi:=\left\{c_{-}=0, \eta=0, h_{3}=c_{+}^{2} / 4\right\}$ (Fig. 2(b)). This emphasizes the relevance of the plasmon-mediated interaction between the emitters, because this scheme lets us switch on/off the collective parameters $\gamma$ and $V$ (Eqs. 7).

Figure 3 also demonstrates that the quantum discord be- comes the 'most relevant' correlation at high laser intensity: $\mathcal{E}_{\mathcal{F}}$ rapidly decays at high intensities and, at about $\ell \sim 1.5 \Gamma$ (see the 'white zone' in graph (b)) the emitters have already experienced collapses, revivals, and early stage disentanglement [37]. In contrast, the discord reaches nonzero stationary values (Fig. 3.a)), indicating the presence of nonclassical correlations different to entanglement.

\section{Detuned molecules}

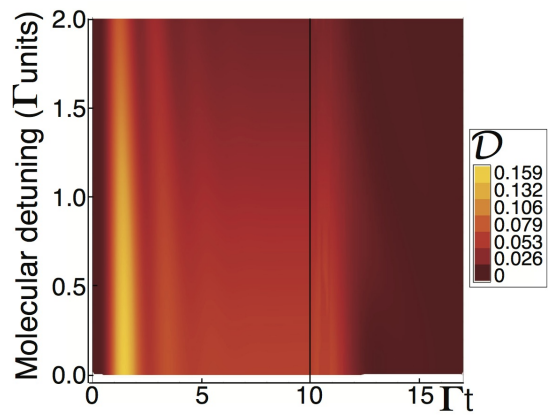

Figure 4: Density plot of the switching mechanism for the quantum discord as function of time and molecular detuning $\delta$ under laser pumping with amplitude $\ell=1.5 \Gamma$, and laser frequency detuning $\Delta=\omega_{L}-\omega_{0}$. Initial state and waveguide parameters are as in Fig. 3 The black vertical line indicates the time at which the laser is turned off.

As yet, we have assumed identical emitters to show the mechanism for switching correlations. We notice, however, that this quantum control also works in a more general physical setup of two molecules with different transition frequencies, $\omega_{1} \neq \omega_{2}$, i.e. with a frequency detuning $\delta=\omega_{1}-\omega_{2}$. In fact, this situation is encountered in any experiment on molecular systems [35, 38].

In this case, the eigenstates of the emitters Hamiltonian are: $|00\rangle,|11\rangle$, and two new entangled states $\left|\phi^{+}\right\rangle=\alpha_{1}|01\rangle+\alpha_{2}|10\rangle$, and $\left|\phi^{-}\right\rangle=\alpha_{2}|01\rangle-\alpha_{1}|10\rangle$, with $\alpha_{1}=\sqrt{V^{2} /\left(\kappa^{2}+V^{2}\right)}, \alpha_{2}=$ $\sqrt{\kappa^{2} /\left(\kappa^{2}+V^{2}\right)}$, and $\kappa=\delta / 2+\sqrt{V^{2}+(\delta / 2)^{2}}$. The new transition frequencies of these eigenstates are, respectively: $\omega_{ \pm}=\omega_{0} \pm$ $\sqrt{V^{2}+(\delta / 2)^{2}}$. The inclusion of a molecular detuning does not allow for a general analytical solution of the master equation (4). If we assume, however, that the emitters have interqubit 
separation $\zeta_{n}$, and are initialized by $c_{-}=0$, in the absence of laser excitation we obtain

$$
\begin{aligned}
z(t) & =2 \eta \mathcal{G}\left[4 V^{2}+\delta^{2} \cos (\Upsilon t)+\mathrm{i} \delta \Upsilon \sin (\Upsilon t)\right] \\
b_{ \pm}(t) & =\mathcal{G}\left[\left(\left(2-c_{+}\right)-p e^{-\Gamma t}\right) \Upsilon^{2} \pm 4 \eta(\cos (\Upsilon t)-1) \delta V\right]
\end{aligned}
$$

where $a(t)$ and $f(t)$ are given as in Eqs. (8), $\mathcal{G}=\exp (-\Gamma t) / 4 \Upsilon^{2}$, and $\Upsilon^{2}=4 V^{2}+\delta^{2}$. In contrast to the previous case of identical molecules, this evolution is not independent of $V$ so the correlations may exhibit oscillatory behaviour due to the terms in $z(t)$. As we are interested in a rapid switching of correlations, we apply the complete configuration $\Xi$ to the initial condition such that the matrix element $z(t)$ goes to zero, and the factor $\Upsilon^{2}$ simplifies in $b_{ \pm}(t)$. The reduction of Eqs. (11) shows that, under the parameters choice $\left\{\Xi, \zeta_{n}\right\}$, the same result as for identical, resonant molecules is recovered: all correlations remain zero.

To understand the influence of the molecular energy mismatch under laser pumping, we plot the quantum discord in terms of $\delta$ in Fig. 4. We illuminate emitter 1 with a laser intensity $\ell=1.5 \Gamma$, and take into account the eigenenergies of the intermediate states to introduce the laser frequency detuning $\Delta:=\omega_{L}-\omega_{0}=\sqrt{V^{2}+(\delta / 2)^{2}}$. This choice makes the dynamical action of the two entangled eigenstates effective, so both the quantum discord and the entanglement can be enhanced. The value reached by the discord slightly decreases as the molecular detuning increases from 0 to $2.0 \Gamma$, because the emitters start to be uncoupled from each other for $\delta \gg V$.

The fact that, for our initial conditions, the interqubit coupling $V$ is maximized for the collective decay rate $\gamma=0$ may be exploited to perform quantum logic gates. For the interqubit dipolar coupling here considered, $\hat{H}_{12}$, one can define an optimal entangling gate for the dipole-dipole interaction; this, plus local unitaries, give a universal gate-set [32]. The plasmonassisted dipolar interaction $V$, for e.g. $\zeta_{1}=3 / 4$, in combination with an applied laser field, would then allow the realization of conditional quantum dynamics. As we have demonstrated, this works for molecules separated by distances of at least $0.4 \mu \mathrm{m}$.

\section{Concluding remarks}

We stress that our approach can be tested in the laboratory with present day technology. The plasmonic structure is modelled with parameters that can be achieved with e.g. either $\Lambda$ or V-groove channel waveguides [39]. Although we note that our calculations are based on ideal plasmonic structures, the basic phenomena that we have theoretically predicted should still be observable in an experiment using real structures. Perylene dyes are suitable single emitters because they are highly photostable, very bright and well characterized at the singlemolecule level [38]. The deterministic placement of single emitters on plasmonic structures [8] can be achieved by lithographic methods or chemical surface functionalization. For the initialization and coherent control of this plasmon-coupled dimer system, (high resolution) laser addressing combined with time-correlated single-photon counting techniques are readily available [35, 38, 36]. These allow the quantification of the molecules' dipolar coupling strength, the decay rates, and photon emission required in the generation of the quantum states and the correlations here described.

It is worth pointing out that for other waveguide geometries, the computed $\beta$-factor can reach values well below those used here. For example, for a cylindrical waveguide the highest predicted $\beta$-factor is $\sim 0.6$ [3, 9]. In such a case, the parameter window for which both the dimer to waveguide surface distance, and the emitter-emitter separation fall within the plasmonic approximation Equations (7) becomes limited, and the losses into the metal, as well as the radiation into vacuum play more significant roles and hence an evaluation of the full Green's tensor is required. This said, the main effect on the results here reported would be a reduction in the degree of quantum correlations in the dimer system, and the protocol for long-distance quantum control here described would continue to work.

In summary, we have demonstrated a mechanism for controlling and switching nonclassical correlations between qubits. Our proposal is based on a set of distant quantum emitters, that are coupled via the interaction with the plasmon modes of a waveguide and driven by an external laser field. We have shown that discord plays a key role in the proposed emitters' conditional quantum dynamics protocol. We have also given a suitable qubit preparation for which the emitters' quantum correlation is always greater than the classical one. Our results could be exploited for quantum computing and communication at long interqubit distances, with the potential for long-range quantum circuitry integration.

\section{Acknowledgements}

C.E.S. thanks the Colombian Administrative Department of Science, Technology and Innovation (Colciencias) for a fellowship. J.H.R. gratefully acknowledges Universidad del Valle for partial funding under grant CI 7930, and for a leave of absence. We thank L. L. Sánchez-Soto for fruitful discussions. R.H. acknowledges the DFG under grant GRK 1640.

\section{Appendix A. Classical and quantum correlations}

The classical correlation is the maximum extractable classical information from a partition, say $A$, when a set of positive operator valued measures (POVMs) has been performed on the other partition $(B)$ [20]. The measurement of classical correlation, as introduced in Ref. [20], is given by:

$$
C\left(\rho_{A B}\right)=\sup _{\left\{\Pi_{j}^{B}\right\}}\left[S\left(\rho_{A}\right)-\sum_{j} p_{j} S\left(\rho_{A \mid \Pi_{j}^{B}}\right)\right],
$$

where $S\left(\rho_{A \mid \Pi_{j}^{B}}\right)$ is the entropy associated to the density matrix of subsystem $A$ after the measure. Such correlation must be non-increasing, and invariant under local unitary operations, and $C\left(\rho_{A B}\right)=0$ if and only if $\rho_{A B}=\rho_{A} \otimes \rho_{B}$.

The set of POVMs that maximize the classical correlation is a complete unidimensional measurement $\left\{\Pi_{j}^{B}\right\}[23]$. If $\{|0\rangle,|1\rangle\}$ defines the basis states for the qubit $B$, the projectors can be 
written as $\Pi_{j}^{B}=\mathbf{1} \otimes|j\rangle\langle j|, j=a, b$, where $|a\rangle=\cos \theta|0\rangle+$ $e^{\mathrm{i} \phi} \sin \theta|1\rangle,|b\rangle=e^{-i \phi} \sin \theta|0\rangle-\cos \theta|1\rangle$, and the optimization is carried out over angles $\theta$ and $\phi$. The measure $C$ is antisymmetric by definition, and, without loss of generality, we take the qubit $B$ to be the one measured.

Following the definition for $C\left(\rho_{A B}\right)$, a simple way to introduce the total quantum correlation in a composite bipartite system is $\mathcal{D}\left(\rho_{A B}\right)=\mathcal{I}\left(\rho_{A B}\right)-C\left(\rho_{A B}\right)$. In terms of the von Neumann entropies, the quantum correlation, which coincides with the definition for the quantum discord given in Ref. [25], reads

$$
\mathcal{D}\left(\rho_{A B}\right)=S\left(\rho_{B}\right)-S\left(\rho_{A B}\right)+\inf _{\left\{\Pi_{j}^{B}\right\}} \sum_{j} p_{j} S\left(\rho_{A \mid \Pi_{j}^{B}}\right)
$$

For pure states, $\mathcal{D}=S\left(\rho_{B}\right)$, and $D=0$ if and only if the system is purely classically correlated.

\section{References}

[1] T. D. Ladd, F. Jelezko, R. Laflamme, Y. Nakamura, C. Monroe, and J. L. O’Brien, Nature 464 (2010) 45.

[2] A. González-Tudela, D. Martín-Cano, E. Moreno, L. Martín-Moreno, C. Tejedor, and F. J. García-Vidal, Phys. Rev. Lett. 106 (2011) 020501.

[3] D. Martín-Cano, A. González-Tudela, L. Martín-Moreno, F. J. GarcíaVidal1, C. Tejedor, and E. Moreno, Phys. Rev. B. 84 (2011) 235306.

[4] W. L. Barnes, A. Dereux, and T. W. Ebbesen, Nature 424 (2003) 824

[5] M. I. Stockman, Phys. Today 64 (2011) 39.

[6] A. V. Akimov et al., Nature 450 (2007) 402.

[7] R. Kolesov et al., Nature Phys. 5 (2009) 470.

[8] A. G. Curto, G. Volpe, T. H. Taminiau, M. P. Kreuzer, R. Quidant, and N. F. van Hulst, Science 329 (2010) 930.

[9] D. Martín-Cano, L. Martín-Moreno, F. J. García-Vidal, and E. Moreno, Nano Lett. 10 (2010) 3129.

[10] R. F. Oulton, G. Bartal, D. F. P. Pile, and X. Zhang, New J. Phys. 10 (2008) 105018.

[11] D. Dzsotjan, J. Kästel, and M. Fleischhauer, Phys. Rev. B 84 (2011) 075419; D. Dzsotjan, A. S. Sorensen, and M. Fleischhauer, Phys. Rev. B 82 (2010) 075427.

[12] W. H. Zurek, Rev. Mod. Phys. 75 (2003) 715.

[13] J. H. Reina, L. Quiroga, and N. F. Johnson, Phys. Rev. A 65 (2002) 032326; C. Wang, and Q.-H. Chen, New J. Phys. 15 (2013) 103020.

[14] R. Hanson, L. P. Kouwenhoven, J. R. Petta, S. Tarucha, and L. M. K. Vandersypen, Rev. Mod. Phys. 79 (2007) 1217; K. Roszak, P. Mazurek, and P. Horodecki, Phys. Rev. A 87 (2013) 062308.

[15] Y. Makhlin, G. Sch'on, and A. Shnirman, Rev. Mod. Phys. 73 (2001) 357; M. Herrera and J. H. Reina, J. Supercod. Nov. Magn. 25 (2014) 2149.

[16] J. M. Raimod, M. Brune, and S. Haroche, Rev. Mod. Phys. 793 (2001) 565; C. Monroe, Nature 416 (2002) 238.

[17] M. Thorwart, J. Eckel, J. H. Reina, P. Nalbach, and S. Weiss, Chem. Phys. Lett. 478 (2009) 234.

[18] C. E. Susa, J. H Reina, and L. L. Sánchez-Soto, J. Phys. B 46 (2013) 224022.

[19] C. E. Susa and J. H. Reina, Phys. Rev. A 85 (2012) 022111.

[20] L. Henderson and V. Vedral, J. Phys. A 34 (2001) 6899.

[21] G. Lindblad, Lecture Notes in Physics, Eds. C. Bendjaballah et al., Vol. 378, 71, Springer, 1991.

[22] B. Groisman, S. Popescu, and A. Winter, Phys. Rev. A. 72 (2005) 032317.

[23] S. Hamieh, R. Kobes, and H. Zaraket, Phys. Rev. A 70 (2004) 052325.

[24] K. Modi, T. Paterek, W. Son, V. Vedral, and M. Williamson, Phys. Rev. Lett. 104 (2010) 080501.

[25] H. Ollivier and W. H. Zurek, Phys. Rev. Lett. 88 (2001) 017901.

[26] S. Luo, Phys. Rev. A 77 (2008) 042303.

[27] A. Datta, A. Shaji, and C. M. Caves, Phys. Rev. Lett. 100 (2008) 050502; V. Madhok and A. Datta, Int. J. Mod. Phys. B 27 (2013) 1245041.

[28] B. P. Lanyon, M. Barbieri, M. P. Almeida, and A. G. White, Phys. Rev. Lett. 101 (2008) 200501.
[29] S. Luo and Q. Zhang, J. Stat. Phys. 136 (2009) 165.

[30] W. K. Wootters, Phys. Rev. Lett. 80 (1998) 2245.

[31] T. Hümmer, F. J. García-Vidal, L. Martín-Moreno, and D. Zueco, Phys. Rev. B 87 (2013) 115419.

[32] J.H. Reina, R. G. Beausoleil, T. P. Spiller, and W. J. Munro, Phys. Rev. Lett. 93 (2004) 250501; C. E. Susa and J. H. Reina, Phys. Rev. A 82 (2010) 042102.

[33] A. González-Tudela, P. A. Huidobro, L. Martín-Moreno, C. Tejedor, and F. J. García-Vidal, Phys. Rev. Lett. 110 (2013) 126801.

[34] U. Fano, Rev. Mod. Phys. 55 (1983) 855; ibid. 29 (1957) 74.

[35] C. Hettich, C. Schmitt, J. Zitzmann, S. Kühn, I. Gerhardt, V. Sandoghdar, Science 298 (2002) 385.

[36] R. Hildner, D. Brinks, and N. F. van Hulst, Nature Phys. 7 (2011) 172.

[37] T. Yu and J. H. Eberly, Science 323 (2009) 598.

[38] E. Lang, R. Hildner, H. Engelke, P. Osswald, F. Würthner, and J. Köhler, Chem. Phys. Chem. 8 (2007) 1487.

[39] S. I. Bozhevolnyi, V. S. Volkov, E. Devaux, J-Y. Laluet, and T. W. Ebbesen, Nature 440 (2006) 508; S. I. Bozhevolnyi, V. S. Volkov, E. Devaux, and T. W. Ebbesen, Phys. Rev. Lett. 95 (2005) 046802. 